\title{
An Investigation on Frequency Selective Antenna Interface Based on Optimization Approach
}

\author{
Hamid Yadegar Amin, Jiangcheng Chen, Markus Berg, Aarno Pärssinen
}

University of Oulu, Finland, Hamid.YadegarAmin@oulu.fi

Keywords: Cellular IoT, RF filter, filter synthesis, impedance matching network, two-port networks, antenna control solution

\begin{abstract}
New long range wireless standards, such as LTE-NB, for Internet-of-Things (IoT) devices have very challenging requirements for power consumption, however, relax, somewhat, linearity requirements of the receiver. Efforts to make low power, low cost and compact devices rise new challenges in the whole level of design including antenna interface which is the entry block of each transceiver chain. High system efficiency and selectivity as close as possible to antenna interface are key design criteria. This issue, demands the designer to ensure the optimality of the antenna interface. This paper presents a precise method for designing two-port impedance matching filters loaded by arbitrary frequency dependent loads. Above mentioned strict constraints favour using fewer number of elements and thus reduce potential losses. A design technique to quickly evaluate all possible topologies from a limited set of elements is presented. The idea is implemented in MATLAB and verified by a design example including lossy elements and practical terminations.
\end{abstract}

\section{Introduction}

Antenna interface filters are essential parts of every RF transceiver. They are supposed to deliver maximum power in a specific bandwidth and suppress out-of-band harmonics which have an important contribution to overall performance including power consumption, noise and linearity [1]. Cellular systems have typically set very strict demands for performance and thus front-end filtering. However, the recent version targeted to long range IoT solutions has significantly relaxed linearity requirement [2-3] and therefore gives opportunity for the relaxed filtering in antenna interface. As early tries, Chebyshev and Butterworth impedance matching filters are investigated with fixed topology and specific loads [4-5]. However, they were impractical in solving the problem for arbitrary frequency dependent terminations. Later on, Carlin developed a CAD model namely real frequency technique (RFT) [6] to solve single matching problem for any type of loads. This method was improved later by Yarman as simplified real frequency technique (SRFT) [7-8]. In SRFT, the solution is sought by approximating input reflection coefficient which is formed as a rational function whose order is set in advance. Therefore, the solution is searched within a specific degree of network which questions optimality of the solution. Also, coefficients of the numerator polynomial are determined through an optimization process by choosing values for the coefficients and synthesizing the acquired impedance function [11]. Therefore, the relation between optimized coefficients and value of synthesized elements totally is dependent on the synthesizing method and applying any limit for value of elements is complicated. Moreover, SRFT can only investigate lossless LC structures and has no functionality for lossy practical situations with limited Q-factors. Although, it has been widely adopted in designing broadband impedance matching networks [9-10], However, an optimal solution will not be guarantee.

In a later try, Valkonen [12] investigates the maximum achievable bandwidth for Chassis antennas based on an optimization method. In the mentioned paper, the available matching topologies were restricted to two-component " $\mathrm{L}$ section" and some selected "three and four-element" matching networks. The selected topologies are undergone an optimization process to obtain the maximum achievable bandwidth for Chassis antennas. However, the selected topologies are only suitable for Chassis Antennas. Also, the design only considers loss-less ideal condition for limited applications.

As explained, limiting the number of components and their values, pave the way for applying a general, straightforward and precise approach to solve lossy and loss-less impedance matching and filtering. The process sweeps all the possible solutions and gives an overall perspective about the optimum topologies and values.

In section 2, the methodology of the implemented technique is discussed in details. Section 3, deals with a design example with lossy elements and practical loads and in section 4, presents a brief conclusion about the proposed method and simulated results.

\section{Parameter Optimization Approach}

Depending on the application requirements, every telecommunication system needs specific properties from an antenna. In this regard, the interface approach should be as generic as possible to provide effective information for all types of antennas.

\section{A. Topology and Components}

Fig. 1 demonstrates a general view of a ladder antenna interface terminated by arbitrary impedances. Each impedance block $\left(Z_{1}, Z_{2}, \ldots, Z_{N}\right)$ demonstrates an impedance function which in general form, as shown in Eq.1, is a frequency dependent complex function. The impedance block can represent a lossy $\left(Z_{i}(f)=\alpha(\mathrm{f}) \pm \mathrm{j} \beta(\mathrm{f})\right)$ or a lossless function $(Z(f)=$ $\pm \mathrm{j} \beta(\mathrm{f}))$. Also, the network can be defined in a general way to 
start or finish with a shunt or series element. For example, if $Z_{1}=0, Z_{N} \neq 0$, the network begins with shunt component and ends with series one. Where $Z_{S}$ is the actual antenna impedance and $Z_{L}$ is the equivalent impedance for transceiver chain.

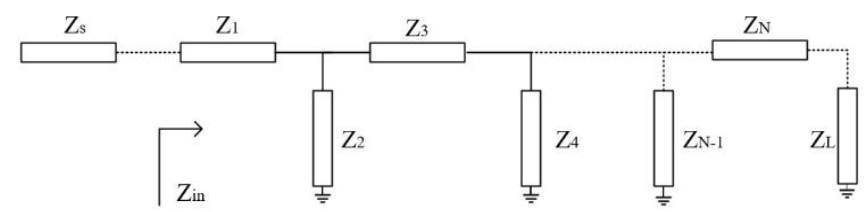

Figure 1:A general view of a ladder impedance network.

$Z_{i}(f)=\left\{\begin{array}{c}\alpha_{1} \pm j \beta_{1}, f=f_{1} \\ \ldots . \\ \alpha_{n} \pm j \beta_{n}, f=f_{n}\end{array}\right.$

Depending on the topology, each of impedance blocks can represent one or a combination of elements such as inductors, capacitors, switches pin diodes, varactor diodes and etc. maximum order of the interest network and their component combination should be defined by user. Fig.2, demonstrates alternatives of topologies when number of impedance blocks are limited to two. In this case, three different topologies can be generated.
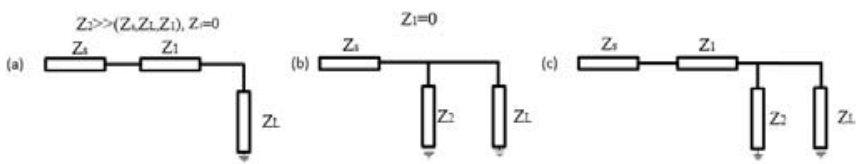

Figure 2:Topology alternatives when the maximum number of impedance blocks is set to two.

Contents of impedance blocks also, are determined by the designer. Fig. 3 presents an example of alternatives for each block.

\begin{tabular}{|c|c|c|c|c|c|}
\hline $\mathrm{zl}$ & $-\infty$ & $\sigma-1$ & sam & $\sigma-H^{m}$ & $\cdots$ \\
\hline $\mathrm{zz}$ & $\Gamma^{+\infty}$ & $3^{\infty}$ & $\underbrace{}_{\substack{\text { varator } \\
\text { Diode }}}$ & & \\
\hline
\end{tabular}

Figure 3:An example of combination of components for each block.

After defining topologies and components' type, numerical values of each impedance block is generated as shown in Eq.1. Depending on the number of frequency points, an impedance vector is defined for each of alternatives. The generated impedance data later are used in optimization process

\section{B. Details on Optimization Procedure}

The process of finding optimal solution accomplishes within two major loops. As, the top level loop, chooses the topology and the inner one investigates proper values of impedances. In each cycle of the inner loop transducer power gain is calculated by Eq.2 [13] and its value compared with the desired gain plot.
$G_{t}=\sqrt{\frac{4 R_{i n} R_{S}}{\left(R_{i n}+R_{S}\right)^{2}+\left(X_{i n}+X_{S}\right)^{2}}}$

Where $R_{\text {in }}$ and $X_{\text {in }}$ are real and imaginary parts of input impedance and $R_{S}$ and $X_{S}$ are real and imaginary values of source impedance.

The goal mask can be one of the standard filter functions such as Chebyshev, Butterworth, Elliptic, Bessel or any other filter mask by designer, enhancing or suppressing only certain frequencies. Afterward, the error value is calculated by subtracting absolute value of both calculated gain graph and the desired mask over the frequency spectrum. The error function can be defined in different manners. In one scenario, it can consider only specific intervals of frequency or, for less sensitive requirements, frequency step size can be decreased which helps to reduce calculation rounds and optimization running time. For specific requirements, error function can be a weighted function giving more weight to error inside selected frequency intervals.

The program exits the inner loop when it covers all possible combination of components for one topology. For each topology, impedance data of states with acceptable error values are sorted for further processes. Having applied the same process for all topologies, they are compared to each other and the results are shown to the designer. Fig.4, depicts the explained design procedure in a flowchart.

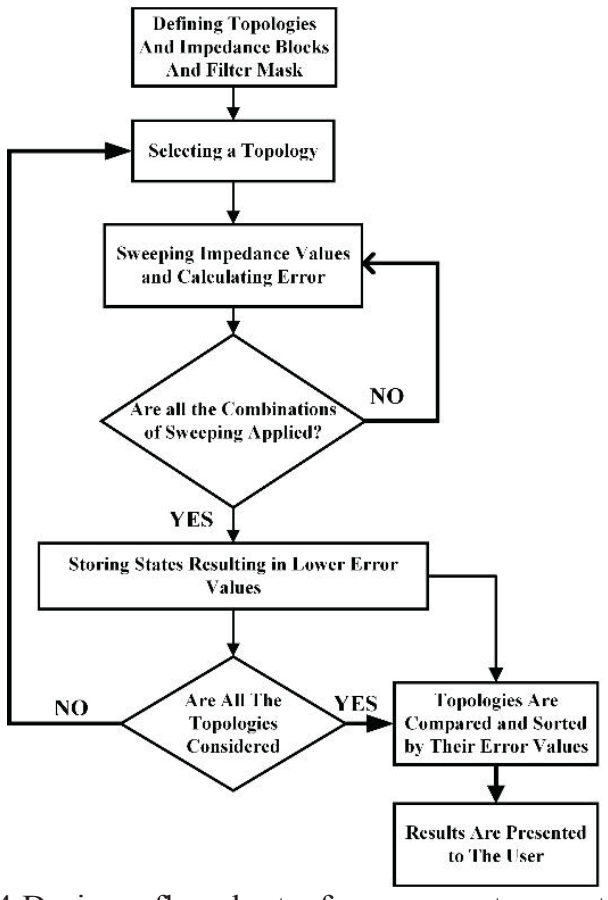

Figure 4:Design flowchart for parameter optimization approach.

\section{Design Example}

In this section the explained algorithm is verified through a design example which is a lossy LC ladder network with maximum 5 impedance blocks. As shown in Fig.5, the network is terminated by two frequency dependent loads which are a 
PIFA antenna and two switches on terminations (PE613010 by Peregrine [14]).

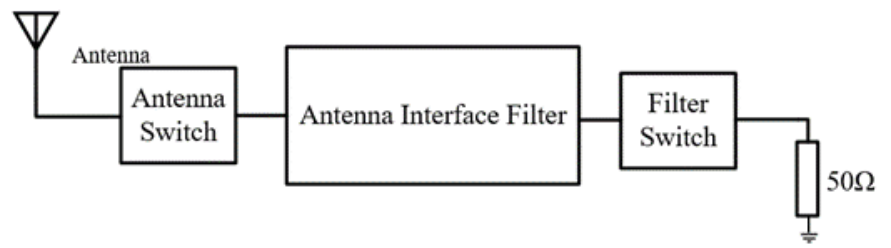

Figure 5:An overall view of the network terminations.

Fig.6, demonstrates a general view of the impedance network which its input impedance is extracted as Eq.3. Where, $Z_{\mathrm{L}}$ and $Z_{s}$ are impedance functions of terminations, $Z_{1}-Z_{5}$ may own inductive, capacitive or resonate function as shown in Fig.7. Therefore, there are 3 possible configurations for each of impedance blocks and the network in its minimum and maximum states can have 1 to 10 components and 363 unique topologies. However, regarding mentioned design requirements, the topologies with more than 5 lumped components are excluded. Therefore, total number of topologies are reduced to 118 .

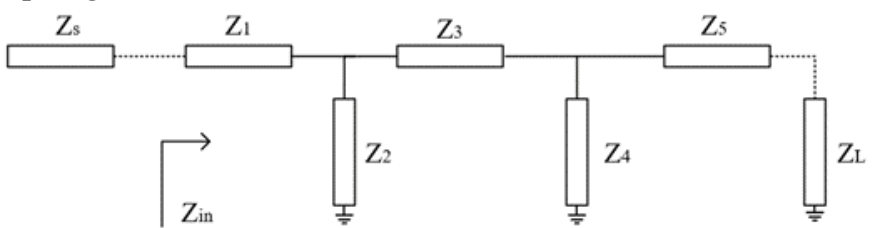

Figure 6:Selected topology of the impedance matching filter.

$$
\operatorname{Zin}=\frac{\begin{array}{c}
Z_{234}+Z_{235}+Z_{23 L}+Z_{24 L}+Z_{245}+Z_{134}+Z_{135}+ \\
Z_{13 L}+Z_{14 L}+Z_{145}+Z_{124}+Z_{125}+Z_{12 L}
\end{array}}{Z_{35}+Z_{3 L}+Z_{4 L}+Z_{45}+Z_{24}+Z_{25}+Z_{2 L}}
$$

\begin{tabular}{|c|c|c|c|}
\hline $\begin{array}{c}\text { Series } \\
\mathrm{Z} \\
\end{array}$ & $\begin{array}{l}\text { State } 1 \\
-1-\end{array}$ & $\begin{array}{l}\text { State 2 } \\
\mathrm{mm}\end{array}$ & State 3 \\
\hline $\begin{array}{l}\text { Shunt } \\
\prod_{\bar{\tau}} z\end{array}$ & $\begin{array}{l}\text { State } 1 \\
\stackrel{\perp}{\rfloor}\end{array}$ & $\stackrel{\text { State } 2}{3}$ & $\stackrel{\text { State 3 }}{\underbrace{}_{\frac{1}{\mp}}}$ \\
\hline
\end{tabular}

Where: $Z_{X Y Z}=Z_{X} Z_{Y} Z_{Z}$

Figure 7:Possible configuration for impedance blocks.

The impedance data of both terminations are imported to MATLAB by their touchstone files. Fig.8, shows input return loss of the used PIFA antenna. For all inductors a same range of values is defined. The range starts from $1.3 \mathrm{nH}$ (LQW15AN minimum value) to $9 \mathrm{nH}$ with step size of $1 \mathrm{nH}$. In a same way, a range of $1 \mathrm{pF}$ to $9 \mathrm{pF}$ with steps of $1 \mathrm{pF}$ is defined for all capacitors. In order to calculate the error, a filter mask is defined which in this case, is a band pass filter with center frequency of $0.850 \mathrm{MHz}$, bandwidth of $15 \mathrm{MHz}$ (LTE Band 20 Uplink) and $8 \mathrm{~dB}$ attenuation at $85 \mathrm{MHz}$ out-of-band. By defining topologies and

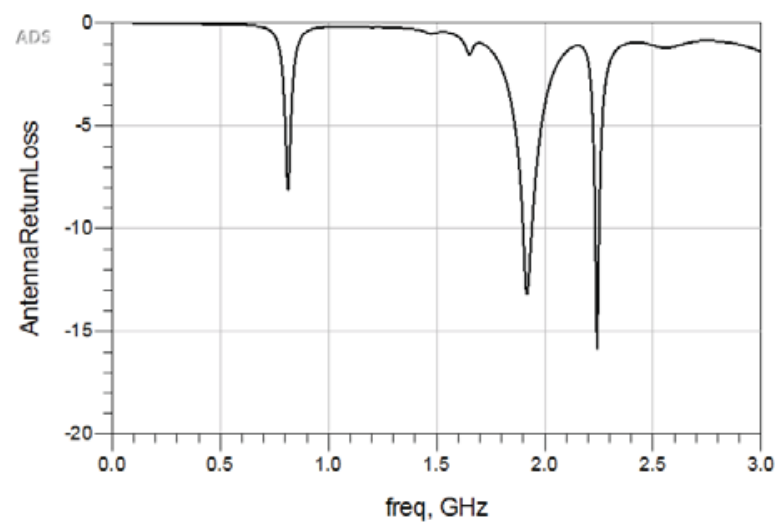

Figure 8:Input return loss of the antenna

values of elements, the program starts to sweep all the possible configurations to find the optimal solution. Fig.9-10, shows four most optimal topologies and their transducer gain performance.

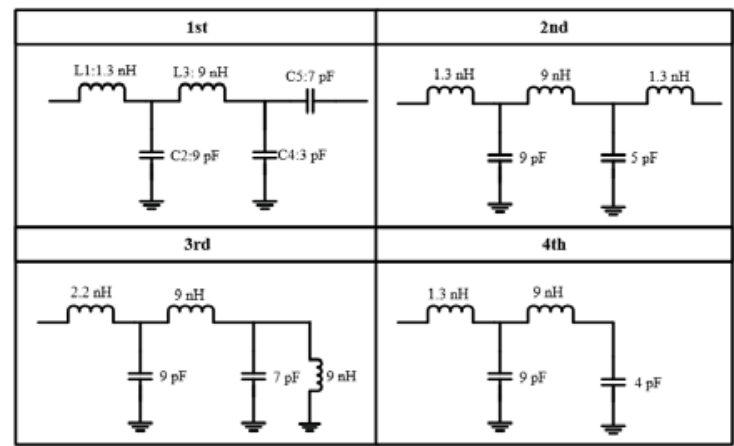

Figure 9:Four most optimal topologies and their values.

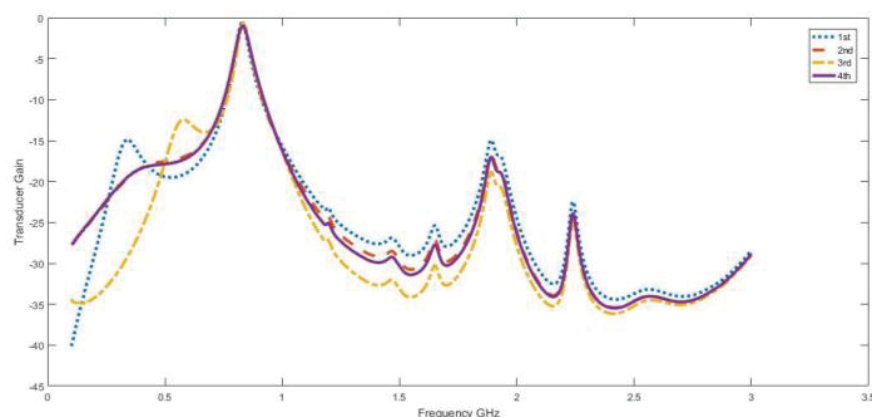

Figure 10:Transducer gain performance of four most optimal topologies.

As explained in section 2, in addition to find the best values for each topology, the program stores other low error states. This process is accomplished by choosing minimum points of error vector for each topology. Fig.11, shows the error plot of first topology shown in Fig.9. As it is seen, the pointed states (state1-state8) cause lower error than others. Therefore, the code stores attributed element values for these states. 
Components' value and their resulted performance for 8 states are presented in Table 1 and Fig. 12 respectively.

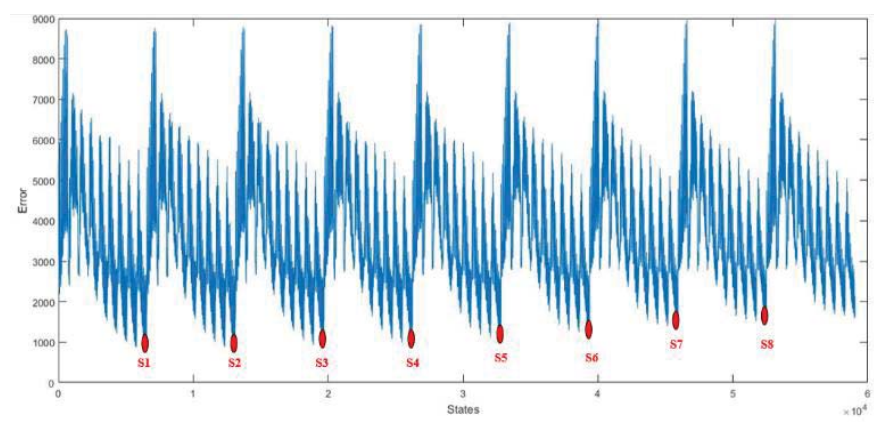

Figure 11:Error analysis for the first topology shown in Fig.7

Table 1: Component value for states cause least amount of error.

\begin{tabular}{|l|lll|l|l|}
\hline State/Element & $\begin{array}{l}\text { L1 } \\
(\mathbf{n H})\end{array}$ & $\begin{array}{l}\text { C2 } \\
(\mathbf{p F})\end{array}$ & $\begin{array}{l}\text { L3 } \\
(\mathbf{n H})\end{array}$ & $\begin{array}{l}\text { C4 } \\
(\mathbf{p F})\end{array}$ & $\begin{array}{l}\text { C5 } \\
(\mathbf{p F})\end{array}$ \\
\hline State 1 & 1.3 & 9 & 9 & 3 & 7 \\
\hline State 2 & 1.3 & 9 & 9 & 3 & 6 \\
\hline State 3 & 4 & 9 & 9 & 3 & 9 \\
\hline State 4 & 5 & 9 & 9 & 4 & 9 \\
\hline State 5 & 6 & 9 & 9 & 4 & 9 \\
\hline State 6 & 7 & 9 & 9 & 4 & 9 \\
\hline State 7 & 8 & 9 & 9 & 5 & 8 \\
\hline State 8 & 8 & 8 & 9 & 3 & 9 \\
\hline
\end{tabular}

As it is seen in Table.1, the best solutions for the chosen topology desire to have highest values for C3, L3 and C5. While C4 takes smaller values and L1 is quite flexible. This process can be accomplished for all topologies which gives a clear idea about required value of impedances to get an optimal solution.

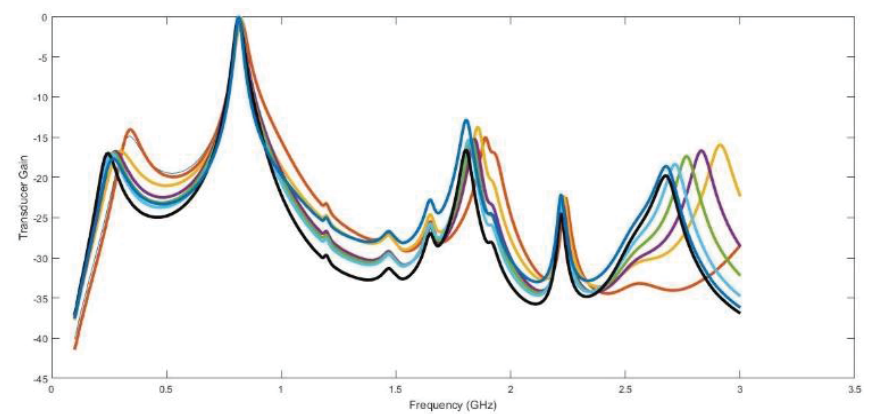

Figure 12: Performance comparison of other best values for first topology in Fig.7

The simulation run time totally depends on the number of investigated states. Using topologies defined in design example, Table.2, compares simulation time for different conditions.
Table 2: Simulation running time for different conditions.

\begin{tabular}{|c|c|c|c|}
\hline $\begin{array}{l}\text { Number of } \\
\text { Lumped } \\
\text { component }\end{array}$ & $\begin{array}{l}\text { Number of } \\
\text { unique } \\
\text { topologies }\end{array}$ & $\begin{array}{l}\text { Resolution of } \\
\text { components } \\
\text { (Same for all) }\end{array}$ & $\begin{array}{l}\text { Simulation } \\
\text { Time }\end{array}$ \\
\hline 3 & 12 & 5 & $3.1 \mathrm{sec}$. \\
\hline 3 & 12 & 10 & $17 \mathrm{sec}$. \\
\hline 4 & 29 & 5 & $33 \mathrm{sec}$. \\
\hline 4 & 29 & 10 & $6 \mathrm{~min}$ \\
\hline 5 & 70 & 5 & $4.7 \mathrm{~min}$ \\
\hline 5 & 70 & 10 & $29 \mathrm{~min}$ \\
\hline
\end{tabular}

As it is seen from Table.2, simulation time increases exponentially by introducing number or resolution of components.

\section{4 conclusions}

An accurate antenna interface solution based on parameter optimization technique is presented. The approach is defined in a general way to be applicable in solving all antenna interface problems. It considers all range of defined component types and Their values to find the optimal solution. Accuracy is proportional to simulation time. However, depending on the application, the simulation time can be reduced by customizing error function. the methodology is explained and a design example is presented.

\section{References}

[1] Ariel Luzzatto, Gadi Shirazi, "Wireless Transceiver Design: Mastering the Design of Modern Wireless Equipment and Systems" John Wiley \& Sons, Ltd, ISBN: 9780470060766, 10 JAN 2007.

[2] 3GPP Technical Specification 36.101. (2016) Evolved Universal Terrestrial Radio Access (E-UTRA); User Equipment (UE) Radio Transmission and Reception. Tech. rep., www.3gpp.org.

[3] 3GPP Technical Specification 36.521. (2016) Evolved Universal Terrestrial Radio Access (E-UTRA); User Equipment (UE) Radio Transmission and Reception. Tech. rep., www.3gpp.org.

[4] W.-K. Chen and T. Chaisrakeo, "Explicit formulas for the synthesis of optimum bandpass butterworth and Chebyshev impedance-matching networks," IEEE Trans. Circuits Syst., vol. 27, pp. 928-942, Oct. 1980.

[5] P. Van der Walt, "On optimum Chebyshev broad-band impedance-matching networks," IEEE Transactions on Circuits and Systems ( Volume: 33, Issue: 10, Oct 1986 ).

[6] H. J. Carlin and P. Amstutz, "On optimum broad-band matching," IEEETrans. Circuits Syst., vol. 28, pp. 401405, May 1981.

[7] Yarman, B.S.; Carlin, H.J. A Simplified "Real Frequency" Technique Applied to Broad-Band Multistage Microwave Amplifiers. IEEE Trans. Microw. Theory Tech. 1982, 30, 2216-2222.

[8] B. S. Yarman and A. Fettweis, "Computer-aided double matching via parametric representation of Brune 
functions," IEEE Trans. Circuits Syst.,vol. 37, pp. 212222, Feb. 1990

[9] Sun, G.; Jansen, R.H. Broadband Doherty Power Amplifier via Real Frequency Technique. IEEE Trans. Microw. Theory Tech. 2012, 60, 99-111.

[10]Wu, D.Y.T.; Mkadem, F.; Boumaiza, S. Design of a Broadband and Highly Efficient 45W GaN Power Amplifier via Simplified Real Frequency Technique. In Proceedings of the 2010 Institute of Electrical and Electronics Engineers (IEEE) MTT-S International Microwave Symposium Digest (MTT), Anaheim, CA, USA, 23-28 May 2010.

[11] Yarman, Binboga Siddik (2008), Design of Ultra Wideband Antenna Matching Networks, Springer, ISBN 978-1-4020-8418-8 p 108-111

[12] Valkonen, Risto, and Anu Lehtovuori. "Determining bandwidth estimators and matching circuits for evaluation of chassis antennas." IEEE Transactions on Antennas and Propagation 63.9 (2015): 4111-4120.

[13]K. Kurokawa, "Power waves and the scattering matrix," IEEE Trans Microw. Theory Tech., vol. 13, pp. 194-202, Mar. 1965.

[14]Peregrine RF switch [Available online] http://www.psemi.com/products/tuning-control-switchestcs/pe613010

[15] Murata Corporation, SMD Lumped Inductors and Capacitors [Website]: http://www.murata.com/ 